\title{
Medullary Thyroid Carcinoma: Do Ultrasonography and F-DOPA-PET-CT Influence the Initial Surgical Strategy?
}

\author{
Lindsay Brammen, MD ${ }^{1}$, Martin B. Niederle, MD $^{2}$, Philipp Riss, MD $^{1}{ }^{(0)}$, Christian Scheuba, MD $^{1}$, \\ Andreas Selberherr, $\mathrm{MD}^{1}$, Georgios Karanikas, $\mathrm{MD}^{3}$, Gerd Bodner, $\mathrm{MD}^{4}$, Oskar Koperek, $\mathrm{MD}^{5}$, and \\ Bruno Niederle, MD ${ }^{1,6}$
}

${ }^{1}$ Section "Endocrine Surgery", Division of General Surgery, Department of Surgery, Medical University Vienna, Vienna, Austria; ${ }^{2}$ Department of Anesthesiology, Medical University Vienna, Vienna, Austria; ${ }^{3}$ Department of Biomedical Imaging and Image-Guided Therapy, Division of Nuclear Medicine, Medical University Vienna, Vienna, Austria; ${ }^{4}$ Department of Biomedical Imaging and Image-Guided Therapy, Division of Radiology, Medical University Vienna, Vienna, Austria; ${ }^{5}$ Department of Pathology, Medical University Vienna, Vienna, Austria; ${ }^{6}$ Former Chief of the Section "Endocrine Surgery", Department of Surgery, Medical University Vienna, Vienna, Austria

\begin{abstract}
Background. At the time of diagnosis, one-third of medullary thyroid carcinoma (MTC) patients show lymph node (LN) or distant metastasis. A metastasized MTC requires different surgical strategies.

Objective. This study aimed to determine the value of ultrasound and [18F]fluoro-dihydroxyphenylalanine positron emission tomography with computed tomography (FDOPA-PET-CT) in localizing MTC, as well as LN and distant metastasis.

Methods. The study included 50 patients (24 males/26 females) with preoperative ultrasound, F-DOPA-PET-CT, and histologically proven MTC. Imaging results were correlated with both preoperative basal calcitonin (bCt) levels and final histology.

Results. Tumors were classified as pT1a:17 (diameter, mean \pm standard deviation: $5.8 \pm 3.0 \mathrm{~mm}), \quad$ pT1b: 15 $(15.0 \pm 3.2 \mathrm{~mm})$, pT2:9 $(27.3 \pm 7.0 \mathrm{~mm})$, and pT3:9 $(38.3 \pm 24.2 \mathrm{~mm})$. The median bCt level was $202 \mathrm{pg} / \mathrm{mL}$ (lower/upper quartile: $82 / 1074 \mathrm{pg} / \mathrm{mL}$ ). Ultrasound was positive for tumor in $45 / 50(92 \%)$ patients $(20.0 \pm$ $16.0 \mathrm{~mm})$ and negative in 5 patients $(3.2 \pm 2.2 \mathrm{~mm})$. Overall, 43/50 (86\%) patients had positive F-DOPA local scans $(20.0 \pm 16.4 \mathrm{~mm})$, while $7(14 \%)$ patients were
\end{abstract}

(C) The Author(s) 2018

First Received: 8 June 2018;

Published Online: 10 October 2018

P. Riss, MD

e-mail: Philipp.riss@meduniwien.ac.at negative $(7.7 \pm 8.1 \mathrm{~mm})$. Lastly, 21/50 (42\%) patients had LN metastasis; $8 / 21$ (38\%) patients had positive LNs suspected with ultrasound, and 12/21 (57\%) patients had positive LNs suspected with F-DOPA. Tumor and LN sensitivity of ultrasound was $92 \%$ and $43 \%$, respectively, and $86 \%$ and $57 \%$ of F-DOPA-PET-CT, respectively. In $3 / 50(6 \%)$ patients and 3/50 (6\%) patients, mediastinal LN metastasis and distant metastasis, respectively, were diagnosed only by F-DOPA-PET-CT.

Conclusion. Ultrasound and F-DOPA-PET-CT are sensitive for the localization of MTC but not for the presence and location of LN metastasis (limitations: size/number). Only F-DOPA ensures the diagnosis of distant metastasis and influences the extent of LN surgery. Surgical strategy cannot be predicted based on neither ultrasound nor F-DOPA-PET-CT.

In medullary thyroid carcinoma (MTC), the number of positive lymph nodes (LNs) and involved anatomical compartments are cancer-specific prognostic factors. ${ }^{1}$ Exact preoperative staging may help to select a stageadapted surgical strategy.

Neck ultrasound is the first imaging modality used in MTC to assess the local extent and regional lymphatic spread. MTC as a neuroendocrine tumor is able to absorb, store, and decarboxylate dopamine. Therefore [18F]fluorodihydroxyphenylalanine positron emission tomography with computed tomography (F-DOPA-PET-CT), 
combining anatomic and 'functional' imaging, may further improve initial local and distal staging of biochemically suspected MTC.

F-DOPA-PET-CT is one of the recommended imaging modalities in patients with persistent MTC and basal calcitonin (bCt) levels $\geq 150 \mathrm{pg} / \mathrm{mL}$ before reoperations; ${ }^{2-5}$ however, no studies with a larger patient population evaluating ultrasound in addition to F-DOPA-PET-CT in locating the primary tumor, $\mathrm{LN}$ and distant metastasis, and possible consequences to the surgical strategy, have been performed.

This prospective observation study aims to determine the value of ultrasound and F-DOPA-PET-CT in radiomorphological detection of primary tumors, identification of possible LN metastasis, and detection of possible distant metastasis before initial surgery, and how a combination of these techniques may help to improve the standardized diagnostic and therapeutic protocol correlating preoperative biochemical and postoperative morphological results.

\section{MATERIALS AND METHODS}

\section{Patients}

Overall, 50 patients (24 males/26 females) with biochemical criteria of MTC $^{6}$ who underwent initial surgery were included in the study. Inclusion criteria consisted of preoperative neck ultrasound and F-DOPA-PET-CT, biochemical parameters, surgery, final histology, and followup in all patients.

The present study was approved by the local Institutional Review Board (EK reference number: 1083/2016). All patients signed written informed consent for all diagnostic and therapeutic procedures.

\section{Laboratory Measurements}

Since 1994, in our institution, the consequent determination of calcitonin ('calcitonin screening') has been performed during the diagnostic work-up of all thyroid nodules regardless of thyroid function, sonomorphology, and size of the thyroid lesion before surgery.

Following the local diagnostic standard operating procedure (SOP), $\mathrm{bCt}>8 \mathrm{pg} / \mathrm{mL}$ for males or $>6 \mathrm{pg} / \mathrm{mL}$ for females, and calcium-stimulated $\mathrm{Ct}>100 \mathrm{pg} / \mathrm{mL}$, were considered highly suspicious of MTC and were therefore an indication for surgery.

The Ct levels were assessed using a two-site chemiluminescent immunometric assay from Diagnostic Products Corporation (Los Angeles, CA, USA), running as a fully automated test on a Siemens Immulite 2000 Immunoassay
System (Siemens Diagnostics) with a lower detection limit of $0.5 \mathrm{pg} / \mathrm{mL}$ and an intra-assay coefficient variation of $\leq 2 \%$.

\section{Diagnostic Procedures}

Neck Ultrasound A standardized neck ultrasound of the cervical and supraclavicular regions (General Electric [GE] LOGIQ E9 device; $9 \mathrm{MHz}$ probe) was performed and analyzed preoperatively by one radiologist (GB), who was aware of the biochemically supposed diagnosis. A suspicious local lesion was characterized as a solid hypoechoic, microcalcification, echogenic foci, intranodular vessel and, if advanced disease, extension beyond the thyroid margin. ${ }^{7}$ Positive metastatic LNs were described as round in shape, necrotic, calcified, loss of the nodal hilum, and peripheral vascularity. ${ }^{8}$

\section{F-DOPA-PET-CT}

As previously described, ${ }^{9}$ PET/CT in a two-dimensional mode with subsequent reconstruction from the top of the skull to the thigh using an integrated camera was performed. One experienced nuclear medicine physician (GK) and one experienced radiologist (GB) interpreted all imaging examinations independently. A lesion was considered 'PET positive' if focal was greater than physiologic tracer uptake and greater than background blood-pool activity. LNs were considered highly suspect for malignancy when the diameter was $>10 \mathrm{~mm}$, and showed round configuration, contrast enhancement, and F-DOPA enhancement without fatty hilus. ${ }^{3}$

True-positive patients had at least one pathologically verified malignant lesion, whereas false-negative patients had positive histopathological results but negative F-DOPA-PET-CT findings.

Surgical Procedure In accordance with our surgical SOP (6), all patients underwent (total) thyroidectomy with bilateral central neck dissection (level VI) and bilateral lateral lymphadenectomy (level II-V) independent of the bCt levels, sparing all vessels, muscles and nerves (functional lateral neck dissection). The surgeon knew all the results of preoperative ultrasound and F-DOPA-PETCT imaging. Only the knowledge of F-DOPA-PET-CTpositive LNs in the mediastinum and distant metastasis influenced the extent of surgery. In patients with positive F-DOPA-PET-CT imaging in the mediastinum, but negative imaging for distant metastasis, a mediastinal LN dissection was conducted. In patients with distant metastasis, a radical central, but a less radical lateral, cervical LN dissection was conducted to avoid complications by positive residual LNs in the central neck. 
Surgical outcome was based on the assessment of bCt (and calcium-stimulated $\mathrm{Ct}$ ) levels determined during the follow-up program. A biochemical cure was achieved if the postoperative $\mathrm{b}$ and stimulated $\mathrm{Ct}$ levels were undetectable.

Pathological Examinations The pathologist was aware of the elevated bCt levels, and therefore of the suspected MTC. Macroscopically suspected lesions (primary tumor; LNs) were stained with hematoxylin and eosin (H\&E), and immunohistochemistry was performed by one pathologist (OK) to confirm primary or metastatic MTC.

TNM Classification TNM classification based on the 7th Edition of the American Joint Committee on Cancer Staging Manual was used for all histopathological results. ${ }^{10}$

\section{Statistical Analysis}

Descriptive statistics analyzed age, sex, tumor characteristics (diameter), number of LNs detected and surgically removed, and bCt levels. Data are given as median (25th75th percentile) or mean ( \pm standard deviation). Sensitivity, specificity, positive predictive value (PPV), and negative predictive value (NPV) were calculated applying Statistical Package for Social Sciences (SPSS) version 23 for Windows (IBM Corporation, Armonk, NY, USA).

Sensitivity for ultrasound and F-DOPA-PET-CT was calculated for different tumor sizes (pT) and different $\mathrm{bCt}$ cut-offs. Patients were grouped into those with tumors $\leq 10 \mathrm{~mm}$ ('microcarcinoma'; pT1a) and those with tumors $>11 \mathrm{~mm}$ ('macrocarcinoma'; pT1b-T4).

Patients with a bCt cut-off level $>43 \mathrm{pg} / \mathrm{mL}$ have MTC independent of the patient's sex. ${ }^{11}$ The American Thyroid Association (ATA) guidelines recommended functional imaging techniques in patients with $\mathrm{bCt}>150 \mathrm{pg} / \mathrm{mL}$, to localize persistent disease. ${ }^{12}$

The optimal cut-off for bCt in diagnosing lateral compartment LN metastasis was calculated using a receiver operating characteristic (ROC) curve (not shown in detail). Positive lateral LNs were only found in patients with bCt levels $\geq 85 \mathrm{pg} / \mathrm{mL}$ (sensitivity $100 \%$ ). Sensitivity, specificity, positive predictive and NPVs to diagnose and locate positive lateral LNs were calculated based on this bCt cutoff.

\section{RESULTS}

Patient characteristics, correlation of tumor classification, and imaging results are summarized in Table 1. Tables 2 and 3 provide the overall results, localizing the primary tumor and affected LNs by ultrasound and F-DOPA-PET-CT.
TABLE 1 Patient characteristics

\begin{tabular}{|c|c|c|}
\hline \multicolumn{2}{|l|}{ No. of patients } & 50 \\
\hline \multicolumn{2}{|c|}{ Males:females } & $24: 26$ \\
\hline \multicolumn{2}{|c|}{ Mean age, years (range) } & $56.9(8.8-78.9)$ \\
\hline \multicolumn{2}{|c|}{ Basal $\mathrm{CT}^{\mathrm{a}}$ (range) } & $202 \mathrm{pg} / \mathrm{mL}(82-1074)$ \\
\hline \multicolumn{2}{|l|}{ CEA (range) } & $12.8 \mu \mathrm{g} / \mathrm{mL}(3.85-52.3)$ \\
\hline \multicolumn{3}{|c|}{ T-stage classification $^{\mathrm{b}}[n(\%)]$} \\
\hline \multicolumn{2}{|l|}{ pT1a } & $17 / 50(34)$ \\
\hline \multicolumn{2}{|l|}{ pT1b } & $15 / 50(30)$ \\
\hline \multicolumn{2}{|l|}{ pT2 } & $9 / 50(18)$ \\
\hline \multicolumn{2}{|l|}{ pT3 } & $9 / 50(18)$ \\
\hline \multicolumn{2}{|c|}{ Multifocal [n (\%)] } & $8 / 50(16)$ \\
\hline \multicolumn{2}{|c|}{ Unilateral versus bilateral } & 2 versus 6 \\
\hline \multicolumn{2}{|c|}{ Hereditary MTC $[n(\%)]$} & $8 / 50(16)$ \\
\hline \multicolumn{2}{|c|}{ Mean tumor size $[\mathrm{mm}( \pm \mathrm{SD})]$} & $18.3( \pm 16.0)$ \\
\hline \multicolumn{2}{|l|}{$\mathrm{N} 1^{\mathrm{c}}[n(\%)]$} & $21 / 50(42)$ \\
\hline $\mathrm{N} 1 \mathrm{a}[n(\%)]$ & Central & $18 / 21(85.7)$ \\
\hline \multirow[t]{2}{*}{$\mathrm{N} 1 \mathrm{~b}[n(\%)]$} & Lateral & $16 / 21(76.2)$ \\
\hline & Mediastinal & $3 / 21(14)$ \\
\hline \multicolumn{2}{|c|}{ Mean number of LNs removed $( \pm \mathrm{SD})$} & $74( \pm 7)$ \\
\hline \multicolumn{2}{|c|}{ Mean number of positive LNs ( \pm SD) } & $6.7( \pm 2)$ \\
\hline \multicolumn{2}{|l|}{$\mathrm{M} 1^{\mathrm{d}}(\%)$} & $3 / 50(6)$ \\
\hline
\end{tabular}

CEA Carcinoembryonic antigen, $M T C$ medullary thyroid carcinoma, $S D$ standard deviation, $L N s$ lymph nodes, UICC Union for International Cancer Control, LNs lymph nodes

${ }^{\mathrm{a} C}$ Calcitonin (Siemens Diagnostic Products Corporation). Normal range: $\mathrm{bCt}: \leq 8 \mathrm{pg} / \mathrm{mL}$ males, $\leq 5 \mathrm{pg} / \mathrm{mL}$ females; CEA: $0-3.8 \mu \mathrm{g} / \mathrm{L}$ $(0-6.5 \mu \mathrm{g} / \mathrm{L}$ in smokers $)$

${ }^{\mathrm{b}}$ UICC $2010^{10}$ ( $p$ : pathological classification). pT1a: $\leq 10 \mathrm{~mm}$; pT1b: 11-20 mm; pT2: 21-40 mm; pT3: $>40 \mathrm{~mm}$ or any size with minimal extrathyroidal extension; pT4: moderately advanced or very advanced disease

${ }^{\mathrm{c}} \mathrm{N} 1$ : regional LN metastasis; N1a: metastasis to level VI (pretracheal, paratracheal, and prelaryngeal/Delphian LNs N1b. Metastasis to unilateral, bilateral, or contralateral cervical (levels I, II, III, IV, or V), retropharyngeal or superior mediastinal LNs (level VII)

${ }^{\mathrm{d}} \mathrm{M} 1$ : distant metastasis

\section{Primary Tumor}

Neck ultrasound was true positive in 45/50 (90\%) patients (mean diameter: $20.0 \pm 16.0 \mathrm{~mm}$ ). In $5(10 \%)$ patients $(3.2 \pm 2.2 \mathrm{~mm}$; $4 / 5$ with bilateral thyroid nodules), the specific malignant nodule was not preoperatively identified in the correct lobe (Table 3).

F-DOPA-PET-CT was true positive in 43/50 (86\%) patients $(20.0 \pm 16.4 \mathrm{~mm})$. When studying the F-DOPA scans for diagnosis of the primary tumors, the preoperative diagnostic scans were false negative in $7(14 \%)$ patients $(7.7 \pm 8.1 \mathrm{~mm} ; 3 / 7 \quad$ with bilateral thyroid nodules) (Table 2). 
TABLE 2 Diagnosis of primary tumor: ultrasound and F-DOPA-PET-CT
TABLE 3 Value of ultrasound and F-DOPA-PET-CT: analysis of patients with central and lateral neck LN metastasis

\begin{tabular}{|c|c|c|c|c|c|c|}
\hline & \multicolumn{3}{|c|}{ Ultrasound } & \multicolumn{3}{|c|}{ F-DOPA-PET-CT } \\
\hline & Yes $(n)$ & No $(n)$ & Sensitivity $(\%)$ & Yes $(n)$ & No $(n)$ & Sensitivity $(\%)$ \\
\hline All (pT1a-pT3) & 45 & 5 & 90 & 43 & 7 & 86 \\
\hline pT1a & 12 & 5 & 71 & 11 & 6 & 65 \\
\hline pT1b & 15 & 0 & 100 & 15 & 0 & 100 \\
\hline pT2 & 9 & 0 & 100 & 8 & 1 & 89 \\
\hline pT3 & 9 & 0 & 100 & 9 & 0 & 100 \\
\hline $\mathrm{pT} 1 \mathrm{a}(\operatorname{maxDM} \leq 10 \mathrm{~mm})$ & 12 & 5 & 71 & 11 & 6 & 67 \\
\hline pT1b-4 (maxDM $>10 \mathrm{~mm})$ & 32 & 0 & 100 & 31 & 1 & 97 \\
\hline \multicolumn{7}{|l|}{ Calcitonin level $^{\mathrm{a}}$} \\
\hline $\mathrm{bCt} \leq 43 \mathrm{pg} / \mathrm{mL}$ & 8 & 1 & 89 & 4 & 5 & 44 \\
\hline $\mathrm{bCt}>43 \mathrm{pg} / \mathrm{mL}^{\mathrm{b}}$ & 41 & 0 & 100 & 39 & 2 & 95 \\
\hline $\mathrm{bCt} \leq 150 \mathrm{pg} / \mathrm{mL}^{\mathrm{c}}$ & 22 & 1 & 96 & 16 & 7 & 70 \\
\hline $\mathrm{bCt}>150 \mathrm{pg} / \mathrm{mL}$ & 27 & 0 & 100 & 27 & 0 & 100 \\
\hline
\end{tabular}

F-DOPA-PET-CT [18F]fluoro-dihydroxyphenylalanine positron emission tomography with computed tomography, $p T$ pathological tumor classification (11), $\max D M$ maximal tumor diameter, $b C t$ basal calcitonin level, $M T C$ medullary thyroid carcinoma

${ }^{a}$ Two-site chemiluminescent immunometric assay: Diagnostic Products Corporation (Los Angeles, CA, USA)

${ }^{\mathrm{b}}$ All patients with $\mathrm{bCt}>43 \mathrm{pg} / \mathrm{mL}$ were shown to have MTC, independent of the patient's sex

${ }^{\mathrm{c}} \mathrm{bCT}$ of $150 \mathrm{pg} / \mathrm{mL}$ is the lowest value recommended in the American Thyroid Association guidelines for performing functional imaging techniques in (persisting) MTC

\begin{tabular}{|c|c|c|c|c|c|c|}
\hline \multirow[t]{2}{*}{ Lymph node metastasis } & \multicolumn{3}{|c|}{ Ultrasound } & \multicolumn{3}{|c|}{ F-DOPA-PET-CT } \\
\hline & Central & Lateral & Overall $^{\mathrm{a}}$ & Central & Lateral & Overall $^{\mathrm{a}}$ \\
\hline True positive $(n)$ & 1 & 9 & 9 & $5^{\mathrm{b}}$ & 12 & 12 \\
\hline True negative $(n)$ & 32 & 33 & 28 & 32 & 34 & 29 \\
\hline False positive $(n)$ & 0 & 1 & 1 & 0 & 0 & 0 \\
\hline False negative $(n)$ & 17 & 7 & 12 & 13 & 4 & 9 \\
\hline Sensitivity (\%) & 6 & 56 & 43 & 28 & 75 & 57 \\
\hline Specificity (\%) & 100 & 97 & 97 & 100 & 100 & 100 \\
\hline PPV (\%) & 100 & 90 & 90 & 100 & 100 & 100 \\
\hline NPV $(\%)$ & 65 & 83 & 70 & 71 & 89 & 76 \\
\hline Accuracy $(\%)$ & 66 & 84 & 74 & 74 & 92 & 82 \\
\hline
\end{tabular}

F-DOPA-PET-CT [18F]fluoro-dihydroxyphenylalanine positron emission tomography with computed tomography, $L N$ lymph node, $P P V$ positive predictive value, $N P V$ negative predictive value

${ }^{\mathrm{a}}$ Evaluation of ALL neck compartments together

${ }^{\mathrm{b}}$ Patients had both positive central and lateral LNs

\section{Lymph Node Metastasis}

LN metastasis was pathologically reported in 21/50 (42\%) patients. Ultrasound and F-DOPA-PET-CT predicted true-positive LN metastasis in 9/21 (42.9\%) patients and 12/21 (57.1\%) patients, respectively. The overall sensitivities of ultrasound and F-DOPA-PET-CT were $43 \%$ and $57 \%$, respectively (Table 3 ).

\section{Compartment Correlation}

Central Neck (Nla; Localized Disease) In terms of diagnosing central LN metastasis, neck ultrasound was true positive in one patient (diameter: $20 \mathrm{~mm}$ ), while 17/18 (94.4\%) patients were false negative $(6.2 \pm 4.87 \mathrm{~mm}$; sensitivity: $6 \%$; specificity: $100 \%$; overall accuracy: $66 \%$ ) (Table 3).

F-DOPA-PET-CT was true positive in 5/18 (27.7\%) patients $(15.0 \pm 5.77 \mathrm{~mm})$, and false negative in $13 / 18$ 
(72.2\%) patients $(4.41 \pm 2.65 \mathrm{~mm}$; sensitivity: $28 \%$; specificity: $100 \%$; overall accuracy: $74 \%$ ).

Lateral Neck and Mediastinum (N1b; Regional Disease) LN metastasis in the lateral neck was found in 16/21 (76.2\%) patients; neck ultrasound was true positive in $9 / 16(56 \%)$ patients $(11.88 \pm 13.31 \mathrm{~mm})$. With one false-positive and seven false-negative patients $(6.93 \pm 6.56 \mathrm{~mm})$, a sensitivity of $56 \%$ and specificity of 97\% was calculated (overall accuracy: 84\%).

In F-DOPA-PET-CT, 12/16 (75\%) scans were true positive $(11.08 \pm 12.08 \mathrm{~mm})$; four $(25 \%)$ were false negative $(5.62 \pm 5.09 \mathrm{~mm})$. No patients were false positive (sensitivity: 75\%; specificity: 100\%; overall accuracy: $92 \%)$.

Upper Mediastinum Additionally, F-DOPA-PET-CT was true positive in three patients with mediastinal LN metastasis. These patients had bCt levels of 12,411, 14,360 , and $14,686 \mathrm{pg} / \mathrm{mL}$, respectively, and none of these patients had distant metastases.

\section{Comparing the Diagnostic Value of bCt and Imaging Techniques}

All 16 patients with $\mathrm{LN}$ metastasis in the lateral neck had bCt levels of $>85 \mathrm{pg} / \mathrm{mL}$ (sensitivity: 100\%); however, given that 21 LN-negative patients had $\mathrm{bCt}$ levels $>85 \mathrm{pg} / \mathrm{mL}$, the specificity was $38 \%$ (Table 4 ).

\section{Number of Metastasis, Sensitivity of Ultrasound and F-DOPA-PET-CT}

The sensitivity for each imaging technique was analyzed for patients with less than $(n=11)$ or more than $10(n=8)$
LN metastasis. The sensitivity of ultrasound or F-DOPAPET-CT in patients with $\leq 10$ positive LNs was $9 \%$ and $27 \%$, respectively, and $80 \%$ and $90 \%$, respectively, for patients with $>10$ positive LNs. Analyzing the individual patients with true-positive F-DOPA-PET-CT and more than 10 positive LNs, the positive LNs were arranged as LN bulks.

\section{Distant Disease}

Three patients (two males, one female; mean age 63.1 years) had distant metastasis diagnosed in F-DOPAPET-CT preoperatively (liver metastasis: $n=2$; bCt values: $1942 \mathrm{pg} / \mathrm{mL}$ and 22,953 pg/mL; lung, liver and bone metastasis: $n=1$; bCt $8397 \mathrm{pg} / \mathrm{mL}$ ). All three patients underwent a palliative surgical approach with thyroidectomy, central neck, and a less extended lateral LN dissection. F-DOPA-PET-CT positively identified all distant metastasis.

\section{Morbidity}

No permanent complications (hypoparathyroidism, paralysis of the recurrent nerve) were documented in the study patients. Temporary hypoparathyroidism was documented in $7 / 50$ (14\%) patients, and temporary paralysis was documented in 4/100 (4\%) nerves at risk. Two patients suffered from unilateral temporary weakness of the accessory nerve and shoulder dysfunction.

\section{Follow-Up}

Table 5 summarizes the biochemical follow-up of the study population. By definition, 34/50 (68\%) patients were cured. In one (4.8\%) patient and in 15 (30\%) patients,
TABLE 4 Biochemical calculation (optimal bCT cut-off for lateral $\mathrm{LN}$ metastasis: $>85 \mathrm{pg} / \mathrm{mL}$ ) and radiological methods (ultrasound or F-DOPA-PETCT) to predict lateral neck LN metastasis

\begin{tabular}{lccc}
\hline Lymph node metastasis in the lateral neck $^{\mathrm{a}}$ & bCt cut-off $>85 \mathrm{pg} / \mathrm{mL}^{\mathrm{b}}$ & Ultrasound & F-DOPA-PET-CT \\
\hline True positive $(n)$ & 16 & 9 & 12 \\
True negative $(n)$ & 13 & 33 & 34 \\
False positive $(n)$ & 21 & 1 & 0 \\
False negative $(n)$ & 0 & $7^{\mathrm{a}}$ & $4^{\mathrm{b}}$ \\
Sensitivity (\%) & 100 & 56 & 75 \\
Specificity (\%) & 38 & 97 & 100 \\
PPV (\%) & 43 & 90 & 100 \\
NPV (\%) & 100 & 83 & 89 \\
Accuracy $(\%)$ & 58 & 84 & 92
\end{tabular}

F-DOPA-PET-CT [18F]fluoro-dihydroxyphenylalanine positron emission tomography with computed tomography, $b C t$ basal calcitonin level, $L N$ lymph node, $P P V$ positive predictive value, $N P V$ negative predictive value

${ }^{a}$ Patients had 1-17 positive lateral LNs

${ }^{\mathrm{b}}$ Lateral lymph node metastasis was only found in patients with bCt levels $\geq 85 \mathrm{pg} / \mathrm{mL}$ 
TABLE 5 Staging and followup of the study population (mean follow-up: $60 \pm 31$ months)

\begin{tabular}{|c|c|c|c|c|c|c|c|}
\hline \multirow[t]{2}{*}{ Stage } & \multirow[t]{2}{*}{$\mathrm{T}$} & \multirow[t]{2}{*}{$\mathrm{N}$} & \multirow[t]{2}{*}{$\mathrm{M}$} & \multicolumn{4}{|c|}{ Number of patients } \\
\hline & & & & Cured $^{\mathrm{a}}$ & Persisting disease ${ }^{\mathrm{b}}$ & Recurrent disease $^{c}$ & Total \\
\hline \multirow[t]{2}{*}{ I } & $1 \mathrm{a}$ & 0 & 0 & 14 & & & 14 \\
\hline & $1 b$ & & & 7 & & & 7 \\
\hline \multirow[t]{2}{*}{ II } & 2 & 0 & 0 & 4 & & 1 & 5 \\
\hline & 3 & & & 3 & & & 3 \\
\hline \multirow[t]{4}{*}{ III } & $1 \mathrm{a}$ & $1 \mathrm{a}$ & 0 & 2 & & & 2 \\
\hline & $1 b$ & & & 1 & & & 1 \\
\hline & 2 & & & 1 & & & 1 \\
\hline & 3 & & & & & & \\
\hline \multirow[t]{5}{*}{ IVA } & $4 a$ & $0 / 1 \mathrm{a}$ & & & & & \\
\hline & $1 \mathrm{a}$ & $1 b$ & 0 & 1 & & & 1 \\
\hline & $1 b$ & & & 1 & 6 & & 7 \\
\hline & 2 & & & & 1 & & 1 \\
\hline & 3 & & & & $5\left(\right.$ died: $\left.2^{\mathrm{d}}\right)$ & & 5 \\
\hline IVB & $4 b$ & Any N & 1 & & & & \\
\hline \multirow[t]{2}{*}{ IVC } & Any $\mathrm{T}$ & Any N & & & $3\left(\right.$ died: $\left.2^{\mathrm{d}}\right)$ & & \\
\hline & & & $n$ & $34(68 \%)$ & 15 (30\%; died: $4.8 \%$ d $)$ & 1 & 50 \\
\hline \multicolumn{8}{|c|}{ TNM, see Edge and Compton ${ }^{10}$ and Wells et al. ${ }^{12}$} \\
\hline \multicolumn{8}{|c|}{$b C t$ Basal calcitonin level } \\
\hline \multicolumn{8}{|c|}{${ }^{\mathrm{a} C u r e d}$ : At the time of follow-up, normal/undetectable bCt levels } \\
\hline \multicolumn{8}{|c|}{${ }^{\mathrm{b}}$ Persisting disease: bCt levels never decreased to normal/undetectable values } \\
\hline \multicolumn{8}{|c|}{${ }^{\mathrm{c}}$ Recurrent disease: Normalization of bCt levels for at least 1 year, than a recurrent increase } \\
\hline
\end{tabular}

recurrent and persisting disease, respectively, were documented. Five $(4.8 \%)$ patients died of progressive MTC.

\section{DISCUSSION}

This study analyses the value of ultrasound and F-DOPA-PET-CT in localizing MTC, and LN and distant metastasis, before initial surgery to select stage-adapted surgery.

Neck ultrasound and F-DOPA-PET-CT achieved an overall sensitivity of $90 \%$ and $86 \%$, respectively, in locating MTC in the correct thyroid lobe. As expected, the sensitivity depends on the size of the tumor and whether it is found as a solitary nodule or in multiple nodules. When localizing microcarcinoma (tumor size $\leq 10 \mathrm{~mm}$ ), the sensitivity for ultrasound and F-DOPA-PET-CT was $71 \%$ and $67 \%$, respectively, and $100 \%$ and $97 \%$, respectively, for macrocarcinoma (tumor size $>10 \mathrm{~mm}$ ). In four patients with multinodular goiter, ultrasound failed to diagnose the malignant micronodules in all patients, while F-DOPA-PET-CT failed in three of the four patients. In the literature, the sensitivity for ultrasound to localize the primary tumor ranges between 27 and 97\%, and the sensitivity of F-DOPA-PET-CT ranges between 65 and $100 \% .^{22-26}$ This current study exhibited high sensitivity in lesion-based ultrasound and F-DOPA-PET-CT. Ni Mhuircheartaigh et al. ${ }^{13}$ reported an average nodule size of at least $15.6 \mathrm{~mm}$ diagnosed in PET-CT. Therefore, size seems an unreliable criterion when it comes to localizing a malignant thyroid tumor. ${ }^{14-16}$ False negatives could also be due to the fact that F-DOPA is not retained for a long period of time in small-sized MTCs., ${ }^{2,17}$

Several studies document that tumor burden positively correlates with bCt levels. $^{13,14}$ In patients with $\mathrm{bCt}>43$ $\mathrm{pg} / \mathrm{mL}$ and $>150 \mathrm{pg} / \mathrm{mL}$, a sensitivity of $100 \%$ was achieved for diagnosing the primary tumor with ultrasound. However, one must take into account that the radiologist may have been biased in the preoperative neck ultrasound examinations, considering that all patients sent for ultrasound met the biochemical criteria for MTC. In addition, the experience of the radiologist also plays a role in the correct diagnosis. F-DOPA-PET-CT for primary tumor showed a sensitivity of $95 \%$ in patients with bCt $>43 \mathrm{pg} /$ $\mathrm{mL}$, and $100 \%$ in patients with $\mathrm{bCt}>150 \mathrm{pg} / \mathrm{mL}$, compared with $44 \%$ and $70 \%$, respectively, in patients with lower bCt levels. Therefore, bCt levels influence the sensitivity of F-DOPA-PET-CT in primary tumor localization.

There is a broad overlap in the aspect of benign and malignant LNs. In this study, a low sensitivity was achieved in diagnosing LN metastasis by ultrasound. While 
the overall sensitivity of $43 \%$ lies within the reported range $\left(29-100 \%^{18-31}\right)$, it is still much lower than the pooled sensitivity in the reviewed literature $(72 \%)$. In terms of diagnosing central LN metastasis, neck ultrasound demonstrated only a $6 \%$ sensitivity. In particular, micrometastasis may be hidden by the thyroid gland and may therefore be missed on ultrasound. However, this study demonstrated a high PPV (100\%), higher than previous studies $\left(77-83 \%^{24,32-34}\right)$.

Ultrasound demonstrated a sensitivity of $56 \%$ and specificity of $97 \%$ in diagnosing lateral LN metastasis. This study demonstrated a high PPV (90\%), similar to previous studies $\left(85-88 \%{ }^{24,32,35}\right)$. Overall accuracy in the central compartment $(66 \%)$ was lower than in the lateral compartment (84\%), in accordance with other studies. ${ }^{29,36,37}$

When analyzing the individual LN compartments, F-DOPA-PET-CT exhibited only $28 \%$ sensitivity and $100 \%$ specificity for the central compartment. A higher sensitivity (75\%) and specificity (100\%) were seen in the lateral compartment. When applying F-DOPA-PET-CT in diagnosing and localizing LN metastasis, an overall sensitivity of $57 \%$ was demonstrated. In patients with bCt $>43 \mathrm{pg} / \mathrm{mL}$ and $>150 \mathrm{pg} / \mathrm{mL}$, a sensitivity of $60 \%$ and $73 \%$, respectively, was achieved. As previously mentioned, the small size of LN metastasis and the short retention time of F-DOPA may affect $\mathrm{LN}$ imaging and therefore the low overall sensitivity.

As shown by Machens and Dralle ${ }^{1}$, surgical cure is possible in patients with $\leq 10 \mathrm{LN}$ metastases. A subanalysis demonstrated a very low sensitivity of $27 \%$ in localizing positive nodes by F-DOPA-PET-CT when applying the cut-off of $\leq 10 \mathrm{LN}$ metastases. This sensitivity increased to $90 \%$ in patients with $\geq 10 \mathrm{LN}$ metastases. Therefore, in patients where only a few, or micro, LN metastases are expected, F-DOPA-PET-CT is not beneficial for diagnostic purposes. Therefore, these findings justify a radical surgical procedure, even in the lateral neck, taking into account possible skip lesions. ${ }^{1}$

A limitation of ultrasound is that $\mathrm{LN}$ metastasis in the mediastinum or distant metastasis cannot be diagnosed; however, F-DOPA-PET-CT demonstrated 100\% sensitivity and specificity in diagnosing positive mediastinal LNs and distant metastasis. According to the current ATA guidelines, ${ }^{12}$ patients with $\mathrm{Ct}$ levels $>500 \mathrm{pg} / \mathrm{mL}$ before the initial operation, or patients with Ct levels $>150 \mathrm{pg} / \mathrm{mL}$ in follow-up should undergo CT imaging with/without PET. ${ }^{12,38}$ All patients with positive LNs in the mediastinum and/or with distant metastasis had bCt values $\geq 500 \mathrm{pg} / \mathrm{mL}$. Given the high sensitivity of F-DOPA-PET-CT with mediastinal LNs and distant metastases, all patients with bCt values $>500 \mathrm{pg} / \mathrm{mL}$ should primarily undergo F-DOPA-PET-CT to diagnose mediastinal LNs or distant metastasis preoperatively.
Patients with positive LNs in the upper mediastinum, but without distant metastasis, may benefit from mediastinal dissection. Furthermore, in patients with distant metastasis, a less extended neck surgery should be considered to avoid local complications and to circumvent complications arising from an extended surgery in the lateral neck.

Generally, the primary workup by ultrasound documents a nodule that is possibly malignant with a clear indication for surgery. F-DOPA-PET-CT improves the results in the central neck from a sensitivity of $6 \%$ to $28 \%$ in respect of diagnosing positive central nodes. However, neither ultrasound nor F-DOPA-PET are helpful in limiting surgery in the central neck because of the overall imprecise radiological localization of the primary tumor and $\mathrm{LN}$ metastasis.

Independent of a positive or negative preoperative imaging study, the primary operation has to meet oncological standards ([total] thyroidectomy; bilateral central [level VI] dissection) in patients who fulfill the biochemical criteria for MTC. One has to keep in mind that the study was performed within a 'calcitonin screening program' documenting pT1a in $17 / 50$ (34\%) patients (diameter $\leq 5 \mathrm{~mm}$ in 6 [25\%] patients), $3(17.6 \%)$ of whom presented with only one or two central LN micrometastases.

Micrometastatic changes in LNs may also influence the low sensitivity in correctly diagnosing and localizing lateral LN metastasis. While the PPV was only $38 \%$, retrospectively, it may be recommended to apply 'functional lateral neck dissection' in at least all patients with $\mathrm{bCt}>85 \mathrm{pg} / \mathrm{mL}$. However, in 21/50 (42\%) patients, the lateral neck dissection revealed negative LNs.

When determining which preoperative examinations to conduct, the advantages and disadvantages for each test must be evaluated. Neck ultrasound is non-invasive, widely available, less expensive than other imaging techniques, and is very safe as no ionizing radiation is used; however, the results are dependent on the radiologist and his/her training and experience. F-DOPA-PET-CT is a functional technique able to provide important anatomical information, especially when it comes to diagnosing distant metastasis. However, its disadvantages include limited availability, high radiation dose (approximately $25 \mathrm{mSv}^{39,40}$ depending on body weight), possible allergic reactions to the contrast medium, and it is expensive.

\section{CONCLUSIONS}

This large, single-center study evaluated how neck ultrasound and whole-body F-DOPA-PET-CT may influence the initial surgical strategy in a center with routine 
measurement of calcitonin in all patients with thyroid nodules. While the sensitivity of ultrasound in diagnosing the primary tumor was very high $(92 \%)$, it was unfortunately quite low (43\%) in detecting the presence and location of LN metastasis. F-DOPA PET-CT also exhibited a good sensitivity of $86 \%$ in detecting the primary tumor, but also a low sensitivity (57\%) when diagnosing the presence and location of LN metastasis. Therefore, the extent of lateral LN surgery cannot be determined, based on neither ultrasound nor F-DOPA-PET-CT. However, the detection of distant metastasis by functioning imaging may help limit the extent of lateral neck and/or mediastinal dissection. Patients who fulfill the biochemical criteria for MTC should undergo a tailored surgical approach.

ACKNOWLEDGMENTS Open access funding provided by Medical University of Vienna.

DISCLOSURE Lindsay Brammen, Martin B. Niederle, Philipp Riss, Christian Scheuba, Andreas Selberherr, Georgios Karanikas, Gerd Bodner, Oskar Koperek, and Bruno Niederle have no conflicts of interest to declare.

OPEN ACCESS This article is distributed under the terms of the Creative Commons Attribution 4.0 International License (http://crea tivecommons.org/licenses/by/4.0/), which permits unrestricted use, distribution, and reproduction in any medium, provided you give appropriate credit to the original author(s) and the source, provide a link to the Creative Commons license, and indicate if changes were made.

\section{REFERENCES}

1. Machens A, Dralle H. Surgical treatment of medullary thyroid cancer. Recent results. Cancer Res. 2015;204:187-205.

2. Archier A, Heimburger C, Guerin C, et al. (18)F-DOPA PET/CT in the diagnosis and localization of persistent medullary thyroid carcinoma. Eur J Nucl Med Mol Imaging. 2016;43:1027-1033.

3. Beheshti M, Pocher S, Vali R, et al. The value of 18F-DOPA PET-CT in patients with medullary thyroid carcinoma: comparison with 18F-FDG PET-CT. Eur Radiol. 2009;19:1425-1434.

4. Koopmans KP, de Groot JW, Plukker JT, et al. 18F-dihydroxyphenylalanine PET in patients with biochemical evidence of medullary thyroid cancer: relation to tumor differentiation. J Nucl Med. 2008;49:524-531.

5. Luster M, Karges W, Zeich K, et al. Clinical value of 18-fluorinefluorodihydroxyphenylalanine positron emission tomography/computed tomography in the follow-up of medullary thyroid carcinoma. Thyroid. 2010;20:527-533.

6. Scheuba C, Kaserer K, Bieglmayer C, et al. Medullary thyroid microcarcinoma recommendations for treatment-a single-center experience. Surgery. 2007;142:1003-1010; discussion 1010.e1-3.

7. Wong KT, Ahuja AT. Ultrasound of thyroid cancer. Cancer Imaging. 2005;5:157-166

8. Ahuja AT, Ying M, Ho SY, et al. Ultrasound of malignant cervical lymph nodes. Cancer Imaging. 2008;8:48-56.

9. Rasul S, Hartenbach S, Rebhan K, et al. [(18)F]DOPA PET/ceCT in diagnosis and staging of primary medullary thyroid carcinoma prior to surgery. Eur J Nucl Med Mol Imaging. Epub 15 May 2018. https://doi.org/10.1007/s00259-018-4045-9.
10. Edge SB, Compton CC. The American Joint Committee on Cancer: the 7th edition of the AJCC cancer staging manual and the future of TNM. Ann Surg Oncol. 2010;17:1471-1474.

11. Niederle MB, Scheuba C, Gessl A, et al. Calcium-stimulated calcitonin: the "new standard" in the diagnosis of thyroid C-cell disease-clinically relevant gender-specific cut-off levels for an "old test". Biochem Med (Zagreb). 2018;28:473-484.

12. Wells SA Jr, Asa SL, Dralle H, et al. Revised American Thyroid Association guidelines for the management of medullary thyroid carcinoma. Thyroid. 2015;25:567-610.

13. Ni Mhuircheartaigh JM, Siewert B, Sun MR. Correlation between the size of incidental thyroid nodules detected on CT, MRI or PET-CT and subsequent ultrasound. Clin Imaging. 2016;40:1162-1166.

14. Bollen EC, Goei R, van't Hof-Grootenboer BE, Versteege CW, Engelshove HA, Lamers RJ. Interobserver variability and accuracy of computed tomographic assessment of nodal status in lung cancer. Ann Thorac Surg. 1994;58:158-162.

15. Gdeedo A, Van Schil P, Corthouts B, Van Mieghem F, Van Meerbeeck J, Van Marck E. Comparison of imaging TNM [(i)TNM] and pathological TNM [pTNM] in staging of bronchogenic carcinoma. Eur J Cardiothorac Surg. 1997;12:224-227.

16. Stomper P. Cancer imaging manual. Philadelphia, PA: Lippincott; 1993. pp. 51-60.

17. Soussan M, Nataf V, Kerrou K, et al. Added value of early $18 \mathrm{~F}$ FDOPA PET/CT acquisition time in medullary thyroid cancer. Nucl Med Commun. 2012;33:775-779.

18. Baatenburg de Jong RJ, Rongen RJ, Lameris JS, Harthoorn M, Verwoerd CD, Knegt P. Metastatic neck disease. Palpation versus ultrasound examination. Arch Otolaryngol Head Neck Surg. 1989;115:689-690.

19. Bruneton JN, Roux P, Caramella E, Demard F, Vallicioni J, Chauvel P. Ear, nose, and throat cancer: ultrasound diagnosis of metastasis to cervical lymph nodes. Radiology. 1984;152:771-773.

20. Gorges R, Eising EG, Fotescu D, et al. Diagnostic value of highresolution B-mode and power-mode sonography in the follow-up of thyroid cancer. Eur J Ultrasound. 2003;16:191-206.

21. Sugitani I, Fujimoto Y, Yamada K, Yamamoto N. Prospective outcomes of selective lymph node dissection for papillary thyroid carcinoma based on preoperative ultrasonography. World J Surg. 2008;32:2494-2502.

22. Shimamoto K, Satake H, Sawaki A, Ishigaki T, Funahashi H, Imai T. Preoperative staging of thyroid papillary carcinoma with ultrasonography. Eur J Radiol. 1998;29:4-10.

23. Jeong HS, Baek CH, Son YI, et al. Integrated 18F-FDG PET/CT for the initial evaluation of cervical node level of patients with papillary thyroid carcinoma: comparison with ultrasound and contrast-enhanced CT. Clin Endocrinol (Oxf). 2006;65:402-407.

24. Ahn JE, Lee JH, Yi JS, et al. Diagnostic accuracy of CT and ultrasonography for evaluating metastatic cervical lymph nodes in patients with thyroid cancer. World J Surg. 2008;32:1552-1558.

25. Roh JL, Park JY, Kim JM, Song CJ. Use of preoperative ultrasonography as guidance for neck dissection in patients with papillary thyroid carcinoma. J Surg Oncol. 2009;99:28-31.

26. Hwang HS, Perez DA, Orloff LA. Comparison of positron emission tomography/computed tomography imaging and ultrasound in staging and surveillance of head and neck and thyroid cancer. Laryngoscope. 2009;119:1958-1965.

27. Lee K, Kawata R, Nishikawa S, Yoshimura K, Takenaka H. Diagnostic criteria of ultrasonographic examination for lateral node metastasis of papillary thyroid carcinoma. Acta Otolaryngol. 2010;130:161-166.

28. Park JS, Son KR, Na DG, Kim E, Kim S. Performance of preoperative sonographic staging of papillary thyroid carcinoma 
based on the sixth edition of the AJCC/UICC TNM classification system. AJR Am J Roentgenol. 2009;192:66-72.

29. Choi JS, Kim J, Kwak JY, Kim MJ, Chang HS, Kim EK. Preoperative staging of papillary thyroid carcinoma: comparison of ultrasound imaging and CT. AJR Am J Roentgenol. 2009;193:871-878.

30. Kessler A, Rappaport Y, Blank A, Marmor S, Weiss J, Graif M. Cystic appearance of cervical lymph nodes is characteristic of metastatic papillary thyroid carcinoma. J Clin Ultrasound. 2003;31:21-25.

31. Gonzalez HE, Cruz F, O'Brien A, et al. Impact of preoperative ultrasonographic staging of the neck in papillary thyroid carcinoma. Arch Otolaryngol Head Neck Surg. 2007;133:1258-1262.

32. Kocharyan D, Schwenter F, Belair M, Nassif E. The relevance of preoperative ultrasound cervical mapping in patients with thyroid cancer. Can J Surg. 2016;59:113-117.

33. Mizrachi A, Feinmesser R, Bachar G, Hilly O, Cohen M. Value of ultrasound in detecting central compartment lymph node metastases in differentiated thyroid carcinoma. Eur Arch Otorhinolaryngol. 2014;271:1215-1218.

34. Kim E, Park JS, Son KR, Kim JH, Jeon SJ, Na DG. Preoperative diagnosis of cervical metastatic lymph nodes in papillary thyroid carcinoma: comparison of ultrasound, computed tomography, and combined ultrasound with computed tomography. Thyroid. 2008;18:411-418.

35. Al-Hilli Z, Strajina V, McKenzie TJ, Thompson GB, Farley DR, Richards ML. The role of lateral neck ultrasound in detecting single or multiple lymph nodes in papillary thyroid cancer. Am J Surg. 2016;212:1147-1153.

36. Lee YJ, Kim DW, Park HK, et al. Pre-operative ultrasound diagnosis of nodal metastasis in papillary thyroid carcinoma patients according to nodal compartment. Ultrasound Med Biol. 2015;41:1294-1300.

37. Lee DW, Ji YB, Sung ES, et al. Roles of ultrasonography and computed tomography in the surgical management of cervical lymph node metastases in papillary thyroid carcinoma. Eur J Surg Oncol. 2013;39:191-196.

38. Pacini F, Castagna MG, Brilli L, Pentheroudakis G; ESMO Guidelines Working Group. Thyroid cancer: ESMO Clinical Practice Guidelines for diagnosis, treatment and follow-up. Ann Oncol. 2012;23 Suppl 7:vii110-119.

39. Brix G, Lechel U, Glatting G, et al. Radiation exposure of patients undergoing whole-body dual-modality 18F-FDG PET/ CT examinations. J Nucl Med. 2005;46:608-613.

40. Huang B, Law MW, Khong PL. Whole-body PET/CT scanning: estimation of radiation dose and cancer risk. Radiology. 2009;251:166-174. 